\title{
Potential Use of Alpha-1 Anti-trypsin in the Covid-19 Treatment
}

\author{
Fernanda Martini ${ }^{1}$, Monica De Mattei ${ }^{2}$, Carlo Contini ${ }^{2}$ and Mauro G. Tognon ${ }^{2 *}$ \\ ${ }^{1}$ Laboraotories of Cell Biology and Molecular Genetics, University of Ferrara, Ferrara, Italy, ${ }^{2}$ Department of Medical Sciences, \\ University of Ferrara, Ferrara, Italy
}

Keywords: SARS-CoV-2, COVID-19, AAT activity, cytokine, inflammation, therapy

\section{INTRODUCTION}

The coronavirus disease-2019 (COVID-19), firstly originated in the city of Wuhan, Hubei Province, People's Republic of China, is due to infection by the severe acute respiratory syndrome coronavirus 2 (SARS-CoV-2) (Guo et al., 2020). SARS-CoV-2 has shown high infectivity, and high mortality associated to respiratory failure from acute respiratory distress syndrome (ARDS), becoming rapidly a worldwide health emergency (Guo et al., 2020). In spite of the several efforts of researchers, the limited knowledge on the disease progression and immunological profile, and the absence of medications or vaccines proven to be effective for treatment or prevention of the SARSCoV-2, lead to the urgent need for efficient and safe therapies, and treatments to limit acute inflammation associated with severe pneumonia and mortality (Mirastschijski et al., 2020). Agents such as potent anti-inflammatory drugs, some antivirals including Remdesevir and, recently, hyperimmune plasma, seem promising, whereas several studies are currently ongoing to test and prove their effectiveness (Guo et al., 2020).

Indeed, approved safe therapies with potential ability to control infection and to prevent respiratory complications may be preferential candidates (Zhou et al., 2020), while the range of proposal drugs is rapidly growing (Scalise and Indiveri, 2020; Ye et al., 2020). This approach may readily permit to identify and use safe drugs, until knowledge on the viral biology will allow to identify specific SARS-CoV-2 drugs and/or vaccines.

\section{ALPHA-1 ANTITRYPSIN (AAT)}

Alpha-1 antitrypsin (AAT) protein is one of the major serum proteins involved in antiinflammatory processes (de Serres and Blanco, 2014). AAT, mainly synthesized in the liver, is released into the bloodstream. AAT is primarily known as a serine protease inhibitor (SERPIN) targeting several enzymes involved in tissue damage/repair, whereas its activity is very high in the lower respiratory tract where it provides over $90 \%$ of the defenses against proteases, mainly neutrophil elastase (NE), protecting healthy tissues from the digestive action of proteolytic enzymes. As an acute phase protein, AAT increases 4-6 fold during infections, inflammation, tissue injury, surgery and late pregnancy (Sanford et al., 1999; Buttenschoen et al., 2001; Ziakas et al., 2011; de Serres and Blanco, 2014).

Further, AAT displays immunomodulatory abilities. It inhibits pro-inflammatory cytokines, while increasing anti-inflammatory mediators (Guttman et al., 2015). Specifically, AAT functions have been linked to interleukin 6 (IL6) signaling (Yuan et al., 2018). Also, AAT has shown antiviral activities in in vitro and in vivo studies (Shapiro et al., 2001; Munch et al., 2007; Wanner et al., 2012). In primary Rhesus monkey kidney cells, AAT inhibited H1N1 influenza virus cell infection, whereas in infected mice AAT decreased the mortality rates and inflammatory cytokines (Wanner et al., 2012). In HIV infection, AAT suppressed viral production in chronically infected monocytes, inhibited HIV-1 entry into a cell line designed to detect viral entry, and reduced HIV-1 replication in human peripheral mononuclear cells (Shapiro et al., 2001; Munch et al., 2007). 
AAT is widely studied in clinical setting due to the existence of congenital genetic defects that reduce its concentrations in the blood (Bornhorst et al., 2007). More than 100 allelic variants of the gene coding for the AAT, called Serine Protease InhibitorA1 (SERPINA1) exist, whereas many of them are associated with reduced circulating protein levels or altered protein activity (Bornhorst et al., 2007). Individuals with AAT deficiency (AATD), in the presence or in the absence of concomitant factors such as smoke, environmental pollutants, and age, have a very high risk of developing pathologies of the respiratory system, such as pulmonary emphysema and chronic obstructive pulmonary disease (COPD) associated to a progressive damage of lung parenchyma (Nuñez et al., 2020).

Additionally, reduced levels of AAT or abnormal AAT proteins have been associated with increased susceptibility to viral infections, such as hepatitis B, hepatitis C, HIV-1, and HTLV-1 infections (Hashemi et al., 2005; Settin et al., 2006; da Silva Ferreira et al., 2014, 2017), and the development of autoimmune and chronic inflammatory diseases, such as diabetes mellitus and panniculitis (Hashemi et al., 2006; de Serres and Blanco, 2014).

\section{ALPHA-1 ANTITRYPSIN THERAPY IN COPD}

Currently, AAT therapy, which is a FDA approved drug, is the only available pharmacological treatment that can slow COPD progression in AATD patients (Griese and Scheuch, 2016; Brantly et al., 2018). COPD is a respiratory disease characterized by persistent respiratory symptoms with significant obstruction of airflow, and increased lung and systemic inflammation (Bradford et al., 2017; Celli and Wedzicha, 2019).

The progression of COPD is associated with increased inflammation of the airway and alveolar wall (Chen et al., 2016). Patients affected by COPD frequently present exacerbations, often triggered by bacterial/viral respiratory infections or viruses, which lead to disease progression through an exaggerated inflammatory response, therefore requiring pharmacological treatments (Xiong et al., 2017). COPD exacerbations are characterized by high neutrophil counts, and high levels of C-reactive protein (CRP), as well as inflammatory cytokines including tumor necrosis factor $\alpha(\mathrm{TNF} \alpha)$, interleukin-1 (IL1), interleukin-8 (IL8) and IL6 (Bradford et al., 2017; Xiong et al., 2017; Nuñez et al., 2020).

Intravenous AAT therapy has been used for the treatment of individuals with AATD and COPD since the late 1980s. Clinical AAT efficacy has been reported showing therapeutic effect on FEV1 and CT lung densitometry in observational or registry studies (Chapman et al., 2009, 2015). Further, AAT therapy impacts on several biological parameters involved in COPD and its progression in AATD patients (Campos et al., 2019). AAT therapy restores the serum protein levels to those of normal subjects, significantly reduces protease activities and inflammation by downregulating several inflammatory markers (Brantly et al., 2018; Campos et al., 2019).
The AAT multiple activities have suggested a potential therapeutic use of AAT for the treatment of several inflammatory and autoimmune diseases beyond COPD, as well as viral diseases including HIV-1 infection, whereby AAT infusion can decrease HIV viral load (Forssmann et al., 2010; Lewis, 2012; Wanner et al., 2012). Therefore, the research on the possible benefits of AAT therapy in other diseases is ongoing, particularly in transplant and type-1 diabetes, as well as in rheumatologic diseases (Lewis, 2012; Marcondes et al., 2016). In preclinical studies, beneficial effects of AAT treatment have been observed in autoimmune disease models, such as rheumatoid arthritis and systemic lupus erythematosus, whereas some clinical studies have investigated AAT treatment in graft vs. host disease (GVHD) in organ transplantation and type 1 diabetes mellitus (Grimstein et al., 2011; Lewis, 2012; Wanner et al., 2012; Marcondes et al., 2016).

\section{DISCUSSION}

In COVID-19 patients, disease severity and high mortality are associated to respiratory failure from ARDS and multiple organ dysfunctions due to an impressive cytokine storm, with significant increased levels of several inflammatory mediators, including IL-6, interferon gamma (INF $\gamma$ ), $\mathrm{TNF} \alpha$, interleukin 17 (IL-17), IL-8 (McElvaney et al., 2020; Pedersen and Ho, 2020). Further, the neutrophil-to-lymphocyte ratio (NLR) in peripheral blood, considered a systemic inflammatory biomarker, is increased in patients with COVID-19 with severe disease compared to those with mild disease and healthy controls (McElvaney et al., 2020). Because elevated levels of IL-6 have been associated to poor prognosis and predictor of mortality, the use of IL6 antagonists has been early proposed for the COVID19 treatment. Recently, the treatment with the monoclonal antibody Tocilizumab, which is currently used for the treatment of rheumatoid arthritis, has been considered an attractive approach for the treatment of COVID-19 (Guaraldi et al., 2020). Clinical trials to study the efficacy and safety of the Tocilizumab monoclonal antibody are ongoing in several Countries, including Italy (Guaraldi et al., 2020). Nevertheless, the validity of the treatment is debated (Arnaldez et al., 2020). In fact, IL-6 is a crucial inflammatory cytokine for the development of antibodies and the activation of $\mathrm{T}$ lymphocytes against infectious agents, and its inhibition could even be deleterious, since it would lower the immune response against the SARS-CoV- 2 (Arnaldez et al., 2020).

To date AAT supplement therapy is largely used to avoid disease exacerbation in AATD COPD patients (Brantly et al., 2018; Campos et al., 2019). In spite of several clinical differences, COVID19 and COPD patients may present similar clinical outcome, such as acute exacerbations, resulting in exaggerated inflammatory response and increased NRL and IL6 levels (Chen et al., 2016; Wang et al., 2020). Interestingly, among common comorbidities in COVID19, Wang et al. have shown that COPD is associated with a 5.9-fold higher risk of progression in patients with COVID-19 (Wang et al., 2020). One explanation could be that the expression of angiotensin-converting enzyme 2 (ACE2), 
which is the host cell receptor for SARS-CoV-2 entry, is increased in COPD patients (Leung et al., 2020).

The potential clinical utility of AAT treatment in COVID19 patients is based on the following considerations: (i) AAT acts as an efficient inhibitor of the host transmembrane protease serine 2 (TMPRSS2) protein receptor (Azouz et al., 2020), which is essential during the initial phase of the SARS-CoV2 infection. Indeed, similar to other coronaviruses, SARS-CoV2 binds its envelope spike (S) protein ligand to the ACE2 receptor for entering into cells (Azouz et al., 2020). The host TMPRSS2 receptor acts by processing the viral $S$ protein, allowing $S$ protein-ACE2 interaction and infection of the host cell. Consequently, the AAT treatment may limit the SARS-CoV2 entry into host cells, which represents the first step of the viral infection; (ii) despite AAT increase in COVID-19 patients, according to its role as an inflammatory acute phase protein, it has been shown that the IL6:AAT ratio is higher in patients with the severe/critical disease than in mild/stable disease (McElvaney et al., 2020). Moreover, in severe/critical COVID-19 patients the increase and reduction of the IL-6:AAT ratio has been associated with a poor outcome and clinical improvement, respectively (McElvaney et al., 2020). Therefore, even though AAT levels are correctly risen in COVID-19 patients, AAT augmentation may be useful to modulate the production and activity of key pro-inflammatory cytokines, while preserving the production of the anti-inflammatory cytokine IL-10 (Guttman et al., 2015); (iii) AATD is a largely under-recognized condition (De Serres et al., 2003). AATD state by itself is not a disease, but a genetic background/predisposition to the development of several diseases. One could speculate that individuals carrying SERPINA1 deficient allelic variants may be at higher risk of SARS-CoV-2 infection than those with wild type SERPINA1, as previously shown for retroviral infections (Hashemi et al., 2005; Settin et al., 2006; da Silva Ferreira et al., 2014, 2017). It is also possible to hypothesize that some SERPINA1 deficient allelic variants could not allow AAT to be sufficiently increased or efficient to counteract SARS-CoV-2 infection, leading to the progressive worsening of the COVID-19 disease. Of note, the Italian register of the AATD reports a higher frequency of cases in northern Italy than in central and southern Italy, with the highest incidence in Lombardy (47\%), which is the northern Italian region counting the highest mortality rate for COVID-19, reaching up 85\% cases (Luisetti et al., 2015; World Health Organization., 2020). This possible association between AATD and COVID-19 deserves to be explored, and might reveal subtypes of COVID-19 patients who could benefit from AAT supplementary therapy. In this view, AAT treatment should be also useful to prevent severe outcome in AATD COVID-19 patients presenting mild symptoms.

Based on these observations, it is reasonable to suggest that AAT treatment, used to slow COPD progression, may be considered in COVID-19 patients. As AAT administration is a FDA-approved drug with a confirmed safety profile, this novel therapeutic potential makes AAT a promising candidate to counteract COVID-19 disease. The use of AAT in the treatment of COVID-19 patients will allow to overcome the limits of the Tocilizumab therapy, which could limit the immune response against SARS-CoV-2 (Arnaldez et al., 2020). Furthermore, while treatment with Tocilizumab is limited to blocking only one proinflammatory pathway (IL6/IL6-R), the administration of AAT could have a broader immunomodulatory and "pro-resolving" effect, increasing the chances of clinical success. In fact, from a biochemical point of view, AAT acts both extracellularly as a protease inhibitor and as a ligand of some membrane receptors, inducing a signal, which modulates the immune response (Guttman et al., 2015). AAT is able: (i) to polarize macrophage cells toward a M2 phenotype, known to be anti-inflammatory and promoter of tissue repair/regeneration programs (Guttman et al., 2016). This activity is significant, since monocyte-macrophages are the main players in the initiation and maintenance of the inflammatory cascade induced by SARS-CoV-2 (Guo et al., 2020); (ii) to neutralize the elastase released by neutrophil granulocytes, also involved in the pathogenesis of lung damage; (iii) to favor the differentiation of T lymphocytes toward the Treg phenotype, endowed with immunosuppressive properties that could contribute the shutdown of the cytokine storm (Baranovski et al., 2015).

Finally, engineered AAT ( $\alpha 1-\mathrm{PDX})$ converted to a furin inhibitor may be a promising antiviral molecule for SARS-CoV-2 (Scott and Sheffield, 2020). The human serine proteinase furin is a proprotein convertase, which processes different pathogens for entering host cell (Shiryaev et al., 2007; Coutarda et al., 2020). Furin-mediated cleavage of viral glycoproteins (gp) facilitates HIV-1, measles, and influenza viral infections (Thomas, 2002). $\alpha 1-P D X$ can block HIV-1 gp160 processing, which is required for cellular invasion, whereas it reduces HIV-1 and measles infectivity in vitro. On this ground, $\alpha 1-\mathrm{PDX}$ can be considered as a potential treatment strategy for COVID-19 (Anderson et al., 1993; Watanabe et al., 1995; Bahbouhi et al., 2000).

In conclusion, due to the known roles of AAT and its current use in clinics (Chapman et al., 2009, 2015; Forssmann et al., 2010; Lewis, 2012; Griese and Scheuch, 2016; Marcondes et al., 2016; Brantly et al., 2018; Campos et al., 2019), AAT treatment may represent a safe drug with potential activity in treating COVID19 affected patients. Safe protocols for treatments, including doses and timely infusions are currently available for COPD patients (Chapman et al., 2009, 2015; Griese and Scheuch, 2016; Brantly et al., 2018; Campos et al., 2019). AAT treatment via aerosol, used in COPD treatment, could be administered in COVID-19 patients as well (Griese and Scheuch, 2016). AAT local instillation or aerosol in humid heat vaporization (40$41^{\circ} \mathrm{C}$ ) in the first phase of COVID-19 might be a powerful strategy for hampering SARS-CoV-2 entering into the host cells by inhibiting host cell receptors, significantly decreasing viral replication, risk of evolution to the more severe clinical pictures, reducing hospitalization and death rate. As recently proposed for Remdesivir, local aerosolisation of AAT with hot-wet humid water vaporization (WHV) at $40-41^{\circ} \mathrm{C}$ could significantly decrease viral replication in hours or 2-3 days at most, in the early stages of respiratory disease (Contini et al., 2020). The amount of product required may be minimal, plausibly reducing disease evolution, patient pain and discomfort, the adverse effects of intravenous administration and social cost.

We suggest that AAT treatment deserves the attention of clinicians for its potential utility in the treatment of COVID-19. Further studies evaluating the 
levels and the activity of AAT in COVID-19 patients presenting cytokine storm or affected by AATD will lead to stratification of patients, assessing whether AAT treatment may be useful as a therapy or to prevent severe outcomes.

\section{REFERENCES}

Anderson, E. D., Thomas, L., Hayflick, J. S., and Thomas, G. (1993). Inhibition of HIV-1 gp160-dependent membrane fusion by a furindirected alpha 1antitrypsin variant. J. Biol. Chem. 268, 24887-24891.

Arnaldez, F. I., O’Day, S. J., Drake, C. G., Fox, B. A., Fu, B., Urba, W. J., et al. (2020). The Society for Immunotherapy of Cancer perspective on regulation of interleukin-6 signaling in COVID-19-related systemic inflammatory response. Version 2. J. Immunother. Cancer. 8:e000930. doi: 10.1136/jitc-2020-000930

Azouz, N. P., Klingler, A., and Rothenberg, M. E. (2020). Alpha 1 antitrypsin is an inhibitor of the SARS-CoV2-priming protease TMPRSS2. bioRxiv [Preprint]. doi: 10.1101/2020.05.04.077826

Bahbouhi, B., Bendjennat, M., Guetard, D., Seidah, N. G., and Bahraoui, E. (2000). Effect of alpha-1 antitrypsin Portland variant (alpha 1-PDX) on HIV-1 replication. Biochem. J. 352, 91-98. doi: 10.1042/bj3520091

Baranovski, B. M., Freixo-Lima, G. S., Lewis, E. C., and Rider, P. (2015). T helper subsets, peripheral plasticity, and the acute phase protein, $\alpha 1$-antitrypsin. Biomed. Res. Int. 2015:184574. doi: 10.1155/2015/184574

Bornhorst, J. A., Calderon, F. R. O., Procter, M., Tang, W., Ashwood, E. R., and Mao, R. (2007). Genotypes and serum concentrations of human alpha1 -antitrypsin 'P' protein variants in a clinical population. J. Clin. Pathol. 60, 1124-1128. doi: 10.1136/jcp.2006.042762

Bradford, E., Jacobson, S., Varasteh, J., Comellas, A. P., Woodruff, P., O’Neal, W., et al. (2017). The value of blood cytokines and chemokines in assessing COPD. Respir. Res. 18:180. doi: 10.1186/s12931-017-0662-2

Brantly, M. L., Lascano, J. E., and Shahmohammadi, A. (2018). Intravenous alpha-1 antitrypsin therapy for alpha-1 antitrypsin deficiency: the current state of the evidence. Chronic Obstr. Pulm. Dis. 6, 100-114. doi: 10.15326/jcopdf.6.1.2017.0185

Buttenschoen, K., Buttenschoen, D. C., Berger, D., Vasilescu, C., Schafheutle, S., Goeltenboth, B., et al. (2001). Endotoxemia and acute-phase proteins in major abdominal surgery. Am. J. Surg. 181, 36-43. doi: 10.1016/S0002-9610(00)00534-1

Campos, M. A., Geraghty, P., Holt, G., Mendes, E., Newby, P. R., Ma, S., et al. (2019). The biological effects of double-dose alpha-1 antitrypsin augmentation therapy. A pilot clinical trial. Am. J. Respir. Crit. Care Med. 200, 318-326. doi: 10.1164/rccm.201901-00100C

Celli, B. R., and Wedzicha, J. A. (2019). Update on clinical aspects of chronic obstructive pulmonary disease. N. Engl. J. Med. 381, 1257-1266. doi: 10.1056/NEJMra1900500

Chapman, K. R., Burdon, J. G., Piitulainen, E., et al. (2015). Intravenous augmentation treatment and lung density in severe alphal antitrypsin deficiency (RAPID): a randomised, double-blind, placebo-controlled trial. Lancet. 386, 360-368. doi: 10.1016/S0140-6736(15)60860-1

Chapman, K. R., Stockley, R. A., Dawkins, C., Wilkes, M. M., and Navickis, R. J. (2009). Augmentation therapy for alphal antitrypsin deficiency: a metaanalysis. COPD. 6, 177-184. doi: 10.1080/15412550902905961

Chen, Y. W., Leung, J. M., and Sin, D. D. (2016). A systematic review of diagnostic biomarkers of COPD exacerbation. PLoS ONE. 11(7):e0158843. doi: 10.1371/journal.pone. 0158843

Contini, C., Gallenga, C. E., Neri, G., Maritati, M., and Conti, P. (2020). A new pharmacological approach based on remdesivir aerosolized administration on SARS-CoV-2 pulmonary inflammation: A possible and rational therapeutic application. Med. Hypotheses. 144, 109876. doi: 10.1016/j.mehy.2020.109876

Coutarda, B., Valleb, C., de Lamballeriea, X., Canardb, B., Seidahc, N. G., and Decrolyb, E. (2020). The spike glycoprotein of the new coronavirus 2019-nCoV contains a furinlike cleavage site absent in $\mathrm{CoV}$ of the same clade. Antiviral Res 176, 104742doi: 10.1016/j.antiviral.2020.104742

\section{AUTHOR CONTRIBUTIONS}

FM proposed the hypothesis. MD wrote the first draft. FM, CC, and MT corrected the draft. FM, MD, CC, and MT wrote the final text. MT supervised the work and submitted the manuscript.

da Silva Ferreira, T. C., Freitas Queiroz, M. A., Argañaraz, G. A., Ishak, R., Vallinoto, A. C. R., and Argañaraz, E. R. (2017). A1AT polymorphisms may be associated with clinical characteristics of retrovirus infections in a mixed ethnic population from the brazilian amazon region. Int. J. Infect. Dis. 65, 67-71. doi: 10.1016/j.ijid.2017.10.002

da Silva Ferreira, T. C., Sampaio, E. P., Arganaraz, G. A., Gondim, M. V., Shapiro, L., and Arganaraz, E. R. (2014). Increased prevalence of the alpha-1antitrypsin (A1AT) deficiency-related S gene in patients infected with human immunodeficiency virus type 1. J. Med. Virol. 86, 23-29. doi: 10.1002/jmv.23759

de Serres, F., and Blanco, I. (2014). Role of alpha-1 antitrypsin in human health and disease. J. Intern. Med. 276, 311-335. doi: 10.1111/joim.12239

De Serres, F. J., Blanco, I., and Fernandez-Bustillo, E. (2003). Genetic epidemiology of alpha-1 antitrypsin in southern Europe: France, Italy, Portugal and Spain. Clin. Genet. 63, 490-509. doi: 10.1034/j.1399-0004.2003.00078.x

Forssmann, W. G., The, Y. H., Stoll, M., Adermann, K., Albrecht, U., Tillmann, H. C., et al. (2010). Short-term monotherapy in HIV-infected patients with a virus entry inhibitor against the gp41 fusion peptide. Sci. Transl. Med. 2:63re3. doi: $10.1126 /$ scitranslmed.3001697

Griese, M., and Scheuch, G. (2016). Delivery of Alpha-1 antitrypsin to airways. Ann. Am. Thorac. Soc. 13 Suppl 4, S346-51. 0.1513/AnnalsATS.201507-469KV

Grimstein, C., Choi, Y. K., Wasserfall, C. H., Satoh, M., Atkinson, M. A., Brantly, M. L., et al. (2011). Alpha-1 antitrypsin protein and gene therapies decrease autoimmunity and delay arthritis development in mouse model. J. Transl. Med. 9:21. doi: 10.1186/1479-5876-9-21

Guaraldi, G., Meschiari, M., Cozzi-Lepri, A., Milic, J., Tonelli, R., Menozzi, M., et al. (2020). Tocilizumab in patients with severe COVID19: a retrospective cohort study. Lancet. Rheumatol. 2, e474-84. doi: 10.1016/S2665-9913(20)30173-9

Guo, G., Ye, L., Pan, K., Chen, Y., Xing, D., Yan, K., et al. (2020). New insights of emerging SARS-CoV-2: epidemiology, etiology, clinical features, clinical treatment, and prevention. Front. Cell Dev. Biol. 8:410. doi: 10.3389/fcell.2020.00410

Guttman, O., Baranovski, B. M., Schuster, R., Kaner, Z., Freixo-Lima, G. S., Bahar, N., et al. (2015). Acute-phase protein $\alpha 1$-anti-trypsin: diverting injurious innate and adaptive immune responses from non-authentic threats. Clin. Exp. Immunol. 179, 161-172. doi: 10.1111/cei.12476

Guttman, O., Freixo-Lima, G. S., Kaner, Z., Lior, Y., Rider, P., and Lewis, E. C. (2016). Context-specific and immune cell-dependent antitumor activities of a1-antitrypsin. Front. Immunol. 7:559. doi: 10.3389/fimmu.2016.00559

Hashemi, M., Alavian, S. M., Ghavami, S., de Serres, F. J., Salehi, M., Doroudi, T., et al. (2005). High prevalence of alpha 1 antitrypsin phenotypes in viral hepatitis B infected patients in Iran. Hepatol. Res. 33, 292-297. doi: 10.1016/j.hepres.2005.09.035

Hashemi, M., Naderi, M., Rashidi, H., and Ghavami, S. (2006). Impaired activity of serum alpha-1-antitrypsin in diabetes mellitus. Diabetes Res. Clin. Pract. 75, 246-8. doi: 10.1016/j.diabres.2006.06.020

Leung, J. M., Yang, C. X., Tam, A., Shaipanich, T., Hackett, T. L., Singhera, G. K., et al. (2020). ACE-2 expression in the small airway epithelia of smokers and COPD patients: implications for COVID-19. Eur. Respir. J. 55:2000688. doi: $10.1183 / 13993003.00688-2020$

Lewis, E. C. (2012). Expanding the clinical indications for $\alpha 1$-antitrypsin therapy. Mol. Med. 18, 957-970. doi: 10.2119/molmed.2011.00196

Luisetti, M., Ferrarotti, I., Corda, L., Ottaviani, S., Gatta, N., Tinelli, C., et al. (2015). Italian registry of patients with alpha-1 antitrypsin deficiency: general data and quality of life evaluation. COPD. 12 Suppl 1, 52-57. doi: 10.3109/15412555.2015.1023393

Marcondes, A. M., Hockenbery, D., Lesnikova, M., Dinarello, C. A., Woolfrey, A., Gernsheimer, T., et al. (2016). Response of steroid refractory acute 
GVHD to $\alpha 1$-antitrypsin. Biol. Blood Marrow Transplant. 22, 1596-1601. doi: 10.1016/j.bbmt.2016.05.011

McElvaney, O. J., McEvoy, N., McElvaney, O. F., Carroll, T. P., Murphy, M. P., Dunlea, D. M., et al. (2020). Characterization of the inflammatory response to severe COVID-19 illness. Am. J. Resp. Crit. Care Med. 202, 812-821. doi: $10.1164 / \mathrm{rccm} .202005-1583$ OC

Mirastschijski, U., Dembinski, R., and Maedler, K. (2020). Lung surfactant for pulmonary barrier restoration in patients with COVID-19 Pneumonia. Front. Med. 7:254. doi: 10.3389/fmed.2020.00254

Munch, J., Standker, L., Adermann, K., Schulz, A., Schindler, M., Chinnadurai, R., et al. (2007). Discovery and optimization of a natural HIV-1 entry inhibitor targeting the gp41 fusion peptide. Cell. 2, 263-275. doi: 10.1016/j.cell.2007.02.042

Nuñez, A., Marras, V., Harlander, M., Mekov, E., Esquinas, C., Turel, M., et al. (2020). Association between routine blood biomarkers and clinical phenotypes and exacerbations in chronic obstructive pulmonary disease. Int. J. Chron. Obstruct. Pulmon. Dis. 15, 681-690. doi: 10.2147/COPD.S24 0720

Pedersen, S. F., and Ho, Y. C. (2020). SARS-CoV-2: a storm is raging. J. Clin. Invest. 2020:137647. doi: 10.1172/JCI137647

Sanford, A. J., Chagani, T., Spinelli, J. J., and Pare, P. D. (1999). Alpha1Antitrypsin genotypes and the acute-phase response to open heart surgery. Am. J. Respir. Crit. Care Med. 159, 1624-1628 doi: 10.1164/ajrccm.159.5.97 11004

Scalise, M., and Indiveri, C. (2020). Repurposing nimesulide, a potent inhibitor of the B0AT1 subunit of the SARS-CoV-2 receptor, as a therapeutic adjuvant of COVID-19. SLAS Discov. doi: 10.1177/2472555220934421. [Epub ahead of print].

Scott, B. M., and Sheffield, W. P. (2020). Engineering the serpin $\alpha 1$ antitrypsin: a diversity of goals and techniques. Protein Science. 29, 856-871. doi: $10.1002 /$ pro.3794

Settin, A., El-Bendary, M., Abo-Al-Kassem, R., and El Baz, R. (2006). Molecular analysis of A1AT (S and Z) and HFE (C282Y and H63D) gene mutations in Egyptian cases with HCV liver cirrhosis. J. Gastrointestin. Liver Dis. $15,131-135$

Shapiro, A., Pott, G. B., and Ralston, A. H. (2001). Alpha-1-antitrypsin inhibits human immunodeficiency virus type 1. FASEB J. 15, 115-122. doi: 10.1096/fj.00-0311com

Shiryaev, S. A., Remacle, A. G., Ratnikov, B. I., Nelson, N. A., Savinov, A. Y., Wei, G., et al. (2007). Targeting host cell furin proprotein convertases as a therapeutic strategy against bacterial toxins and viral pathogens. J. Biol. Chem. 282, 20847-20853. doi: 10.1074/jbc.M703847200
Thomas, G. (2002). Furin at the cutting edge: from protein traffic to embryogenesis and disease. Nat. Rev. Mol. Cell Biol. 3, 753-766. doi: 10.1038/nrm934

Wang, D., Hu, B., Hu, C., Zhu, F., Liu, X., Zhang, J., et al. (2020). Clinical characteristics of 138 hospitalized patients with 2019 novel coronavirus-infected pneumonia in Wuhan, China. JAMA. 323, 1061-1069. doi: 10.1001/jama.2020.1585

Wanner, A., Arce, A. D., and Pardee, E. (2012). Novel therapeutic uses of alpha-1 antitrypsin: a window to the future. COPD. 9, 583-588. doi: 10.3109/15412555.2012.717125

Watanabe, M., Hirano, A., Stenglein, S., Nelson, J., Thomas, G., and Wong, T. C. (1995). Engineered serine protease inhibitor prevents furincatalyzed activation of the fusion glycoprotein and production of infectious measles virus. J. Virol. 69, 3206-3210. doi: 10.1128/JVI.69.5.3206-3210.1995

World Health Organization (2020). Coronavirus Disease 2019 (COVID-19) Situation Report. World Health Organization.

Xiong, W., Xu, M., Zhao, Y., Wu, X., Pudasaini, B., and Liu, J. M. (2017). Can we predict the prognosis of COPD with a routine blood test? Int. J. Chron. Obstruct. Pulmon. Dis. 12, 615-625. doi: 10.2147/COPD.S124041

Ye, Q., Wang, B., and Mao, J. (2020). The pathogenesis and treatment of the 'Cytokine Storm' in COVID-19. J. Infect. 80, 607-613. doi: 10.1016/j.jinf.2020.03.037

Yuan, Y., DiCiaccio, B., Li, Y., Elshikha, A. S., Titov, D., Brenner, B., et al. (2018). Anti-inflammaging effects of human alpha-1 antitrypsin. Aging Cell. 17:e12694. doi: 10.1111/acel.12694

Zhou, Y., Hou, Y., Shen, J., Huang, Y., Martin, W., and Cheng, F. (2020). Networkbased drug repurposing for novel coronavirus 2019-nCoV/SARS-CoV-2. Cell Discov. 6:14. doi: 10.1038/s41421-020-0153-3

Ziakas, A. G., Koskinas, K. C., Souliou, E., Gavrilidis, S., Giannoglou, G. D., Gemitzis, K., et al. (2011). Serial measurements of acute phase proteins in patients with acute coronary syndrome. Hellenic J. Cardiol. 52, 293-298.

Conflict of Interest: The authors declare that the research was conducted in the absence of any commercial or financial relationships that could be construed as a potential conflict of interest.

Copyright (C) 2020 Martini, De Mattei, Contini and Tognon. This is an open-access article distributed under the terms of the Creative Commons Attribution License (CC $B Y)$. The use, distribution or reproduction in other forums is permitted, provided the original author(s) and the copyright owner(s) are credited and that the original publication in this journal is cited, in accordance with accepted academic practice. No use, distribution or reproduction is permitted which does not comply with these terms. 